\title{
Super star clusters in the Blue Dwarf Galaxy UM 462^
}

\author{
L. Vanzi ${ }^{\star \star}$ \\ ESO - European Southern Observatory, Alonso de Cordova, 3107 Santiago, Chile
}

Received 17 April 2003 / Accepted 19 June 2003

\begin{abstract}
I present optical observations of the Blue Compact Dwarf Galaxy UM 462. The images of this galaxy show several bright compact sources. A careful study of these sources has revealed their nature of young Super Star Clusters. The ages determined from the analysis of the stellar continuum and $\mathrm{H} \alpha$ are between few and few tens Myr. The total star formation taking place into the clusters is about $0.05 M_{\odot} / \mathrm{yr}$. The clusters seem to be located at the edges of two large round-like structures, possibly shells originated in a previous episode of star formation. The sizes of the shells compare well with the ages of the clusters. Evidence for the presence of an evolved underlying stellar population is found.
\end{abstract}

Key words. galaxies: individual: UM 462 - galaxies: dwarf - galaxies: star clusters

\section{Introduction}

The galaxy UM 462 (UGC 06850) is a Blue Compact Dwarf (BCD) of relatively low metallicity. Low resolution spectroscopy of this object was obtained by the objective prism survey of MacAlpine et al. (1983), higher quality data were collected later on by Terlevich et al. (1991). From these spectra Masegosa et al. (1994) derived a metallicity $12+\log (\mathrm{O} / \mathrm{H})=$ 7.98. More recent observations by Izotov \& Thuan (1998) gave $12+\log (\mathrm{O} / \mathrm{H})=7.95$, that is about $1 / 9$ solar. A radio HI map has been obtained by van Zee et al. (1998), they derive a neutral hydrogen mass of $2.65 \times 10^{8} M_{\odot}$. UM 462 has not been detected in the millimetric CO (Gondhalekar et al. 1998). The most recent optical images available are those of Cairos et al. (2001), they show a quite compact object, about $20 \times 10 \mathrm{arcsec}$ in size, with a double peaked morphology and blue colors, $B-V=0.6-0.3, V-R=0.3, V-I=0.2-0.4$. No further details of the morphology of the galaxy can be inferred from these images due to the low angular resolution. The galaxy is classified as a peculiar BCD in the Near-IR Galaxy Morphological Atlas of Jarret (2000). Vanzi et al. (2002) identify, for the first time, a complex morphology in UM 462. Their near-infrared images reveal the presence of at least 6 young compact clusters that, according to the colors and luminosities observed were tentatively classified as Super Star Clusters (SSC). This discovery and the lack of high quality optical images motivated the work presented here. The paper is organized as follow; in Sect. 2 the new observations are described, Sect. 3 is dedicated to the description of color-color diagrams, Sect. 4 to the properties of the clusters: extinction, age, luminosity, mass, size and morphology. The conclusions are summarized in Sect. 5 .

* Based on observations obtained at the ESO $3.6 \mathrm{~m}$ telescope of La Silla.

$\star \star$ e-mail: lvanzi@eso.org

\section{Observations}

The observations presented in this paper have been obtained on April 31, 2002 at the ESO $3.6 \mathrm{~m}$ telescope of La Silla using the optical imager spectrometer EFOSC-2. Images in the $U, V, R$ and gunn- $i$ broad band filters were obtained with total integration times of 1800, 1350, 900 and 1200 s respectively. Each image was divided in three exposures of equal duration. The data reduction has followed the standard steps, fringes had to be removed from the gunn- $i$ images using sky frames. The photometric calibration was performed using a photometric field of Landolt (1992). A transformation from gunn- $i$ to $I$, available at the telescope, has been applied. The most prominent feature of the images is the presence of several compact sources. The photometry of the sources detected has been obtained over apertures of $1^{\prime \prime}$ in radius. The contribution of the galaxy has been measured on an anulus around each source and subtracted. For each image the photometry has been carried out independently, then values corresponding to the same filter have been combined, the dispersion of the measures gives an estimate of the photometric errors that are always between 0.01 and 0.02 mag. The results of the photometry are presented in Table 1 . In the last column, indicated as $G$, the photometry of the entire galaxy, obtained with a radius of $20^{\prime \prime}$, is reported, the values are in full agreement with those of Cairos et al. (2001).

Spectroscopy has been obtained with the grism \#9 of EFOSC-2 covering the spectral range 4700-6700 $\AA$ with a resolution $R=900$ and a 1 arcsec wide slit. Two spectra were observed at position angles which allowed to include 5 of the compact sources visible in the images. The orientations of the slits and the clusters detected are indicated in Fig. 1 on the $R$ image of the galaxy. The reduction of the spectra followed the standard steps, 1D spectra centered on the compact sources were extracted with an aperture of 2 arcsec. The flux calibration 
Table 1. Aperture photometry $\left(r=1^{\prime \prime}\right)$ of the clusters in UM 462 and corrections for the contribution of the emission lines. The clusters are indicated with the nomenclature given in Fig. 1. $G$ indicates the photometry of the whole galaxy obtained with a radius of $20^{\prime \prime}$.

\begin{tabular}{cccccccccc}
\hline \hline & 1 & 2 & 3 & 4 & 5 & 6 & 7 & 8 & $G$ \\
\hline$U$ & 17.78 & 18.14 & 17.46 & 19.00 & 18.93 & 18.64 & 18.79 & 18.87 & 14.64 \\
$V$ & 17.64 & 18.77 & 18.10 & 19.38 & 19.25 & 18.99 & 19.35 & 19.56 & 14.50 \\
$R$ & 17.95 & 18.68 & 18.19 & 19.28 & 19.05 & 19.17 & 19.29 & 19.56 & 14.23 \\
$I$ & 18.32 & 18.77 & 18.23 & 19.08 & 18.77 & 19.31 & 19.49 & 19.83 & 14.00 \\
$\Delta U$ & 0.13 & 0.07 & 0.05 & 0.02 & 0.01 & 0.06 & 0.07 & 0.05 & - \\
$\Delta V$ & 0.62 & 0.38 & 0.26 & 0.10 & 0.05 & 0.35 & 0.38 & 0.26 & - \\
$\Delta R$ & 0.37 & 0.20 & 0.15 & 0.04 & 0.02 & 0.19 & 0.20 & 0.15 & - \\
$\Delta I$ & 0.00 & 0.00 & 0.00 & 0.00 & 0.00 & 0.00 & 0.00 & 0.00 & - \\
\hline
\end{tabular}

Table 2. Emission lines detected in the clusters. For the sources indicated by $(*)$ the $\mathrm{H} \alpha$ fluxes have been measured from the narrow band image. The fluxes are given in $10^{-15} \mathrm{erg} / \mathrm{s} / \mathrm{cm}^{2}$.

\begin{tabular}{cccccccccccc}
\hline \hline & 1 & 2 & 3 & $4(*)$ & 5 & 6 & $7(*)$ & $8(*)$ & $\mathrm{a}(*)$ & $\mathrm{b}(*)$ & $\mathrm{c}(*)$ \\
\hline $\mathrm{H} \beta$ & 22.5 & 8.7 & 8.4 & - & 0.5 & 6.9 & - & - & - & - & - \\
{$[\mathrm{OIII}]$} & 51.0 & 14.8 & 15.8 & - & 0.8 & 12.0 & - & - & - & - & - \\
{$[\mathrm{OIII}]$} & 153.0 & 44.1 & 46.9 & - & 2.2 & 36.2 & - & - & - & - & - \\
$\mathrm{HeI}$ & 3.9 & 2.4 & 2.0 & - & 1.3 & 2.5 & - & - & - & - & - \\
$\mathrm{H} \alpha$ & 80.9 & 29.0 & 24.9 & 5.6 & 2.1 & 24.8 & 18.3 & 11.9 & 20.6 & 16.4 & 5.4 \\
{$[\mathrm{NII}]$} & 1.5 & 0.7 & 0.4 & - & 0.1 & 0.7 & - & - & - & - & - \\
{$[\mathrm{SII}]$} & 0.8 & - & - & - & - & - & - & - & - & - & - \\
\hline
\end{tabular}

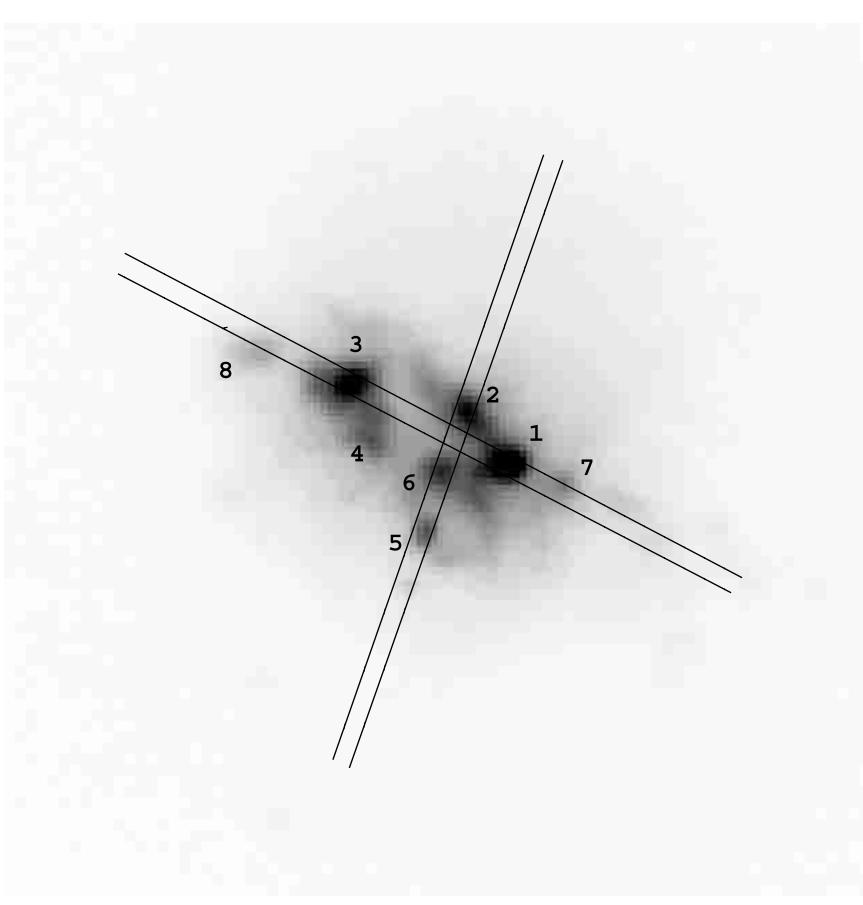

Fig. 1. Image of UM 462 in $R$ with the clusters indicated by numbers and the slit positions used for the spectroscopic observations. North is up, east to the left. The field of view is about $30 \times 30^{\prime \prime}$.

of the spectra has been obtained using the spectro-photometric standard star EG-274 (Hamuy et al. 1992, 1994) observed with a 5 arcsec wide slit and, in a completely independent way, using the photometry extracted from the $V$ image. The two methods gave results consistent within $0.1 \mathrm{mag}$. The spectrum extracted from source 1 is shown in Fig. 2. Several lines typical of HII galaxies are easily identified, the fluxes of the lines detected

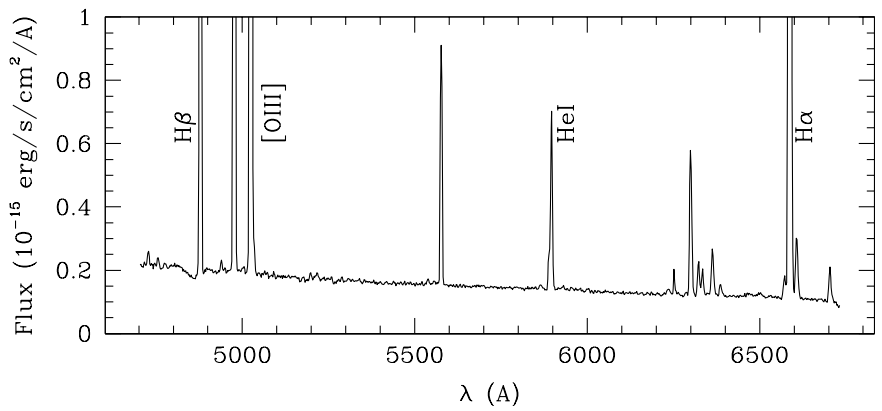

Fig. 2. Optical spectrum of Cluster 1. The emission lines not labeled at 5577 and around $6300 \AA$ are from the sky.

are listed in Table 2. In addition one image in the $\mathrm{H} \alpha$ narrow band filter has been observed with $2700 \mathrm{~s}$ of integration. The width of the filter is $62 \AA$ allowing the $\mathrm{H} \alpha$, redshifted by about $1000 \mathrm{~km} \mathrm{~s}^{-1}$, to be covered. The contribution of the continuum has been subtracted using the broad-band $R$ image rescaled by a suitable factor. The image has been flux calibrated using the $\mathrm{H} \alpha$ fluxes measured in the spectra. In this way it has been possible to have $\mathrm{H} \alpha$ fluxes for the sources not covered by the spectroscopic observations, see Table 2 . The accuracy in this case has been evaluated to be of about $10 \%$. Quite interestingly bright sources that are not prominent in any of the broadband images have been detected in $\mathrm{H} \alpha$, these are indicated with $\mathrm{a}, \mathrm{b}$ and $\mathrm{c}$ in Fig. 3.

\section{The color-color diagrams}

The sources observed have been studied first comparing their colors with the evolutionary tracks produced in color-color diagrams by a single stellar population model. To study the 


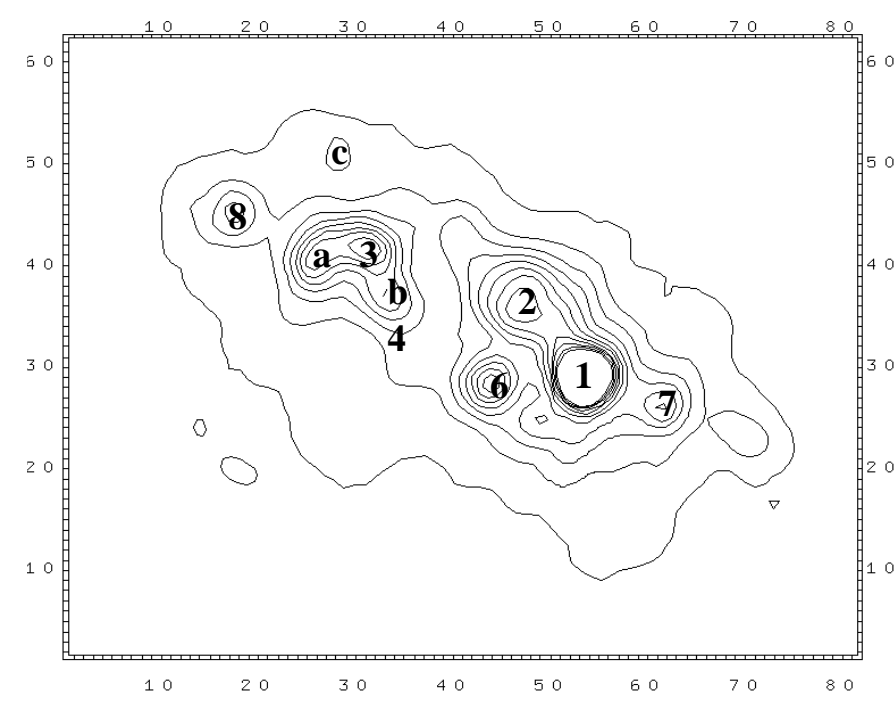

Fig. 3. Contour image of UM 462 in $\mathrm{H} \alpha$. The positions of the clusters are indicated by numbers, new sources not visible in the broad band images are indicated by letters. North is up, east to the left. The field of view is $25 \times 19^{\prime \prime}$.

stellar component through this method it is critical to evaluate any contribution of non stellar origin and to correct for it. The main non stellar contribution to the broad band magnitudes comes, in our case, from the gas. The model Starburst99 (SB99-Leitherer et al. 1999) has been used as a reference, this model includes the effect of the nebular continuum so that it has been sufficient to correct the observations for the contribution of the emission lines. This can be done directly through the observations. Unfortunately the spectra observed do not cover the entire spectral range which includes the photometric filters used, so that a direct measure of all emission lines affecting the photometry has not been possible. Instead I have used the template spectrum of a typical BCD galaxy (Saviane 2003, private communication) rescaled and diluted to match the characteristics of the spectra available for UM 462 in the region of overlap. In particular it has been imposed that the $E W$ of $\mathrm{H} \alpha$ and the $V$ magnitude were the same as those observed. These rescaled spectra have been used to measure the contribution of the emission lines to the photometry. Of course this approach must be considered as not very accurate since the flux emitted in most of the lines of interest depends on the temperature, density and abundance; parameters that have not been matched perfectly, however the method gives a very good idea of the effect and it is certainly the best we can do with the data available. For those sources not observed in spectroscopy the $\mathrm{H} \alpha$ flux obtained from the narrow band image has been used, the corrections in these cases must be considered less accurate. The corrections to the photometry calculated in this way are listed in Table 1. The effect is most important in $V$ while it is always negligible in I due to the absence of important emission lines. In Figs. 4 and 5 color-color diagrams for the clusters in UM 462 are shown. Both the observed (solid circles) and the emission-line-corrected points (solid triangles) are plotted. The errors on the colors are 0.03 at most. The tracks generated by SB99 for an instantaneous burst with solar neighborhood IMF (1-100 $\left.M_{\odot}, 2.35\right)$ and $1 / 5 Z_{\odot}$ abundance are plotted

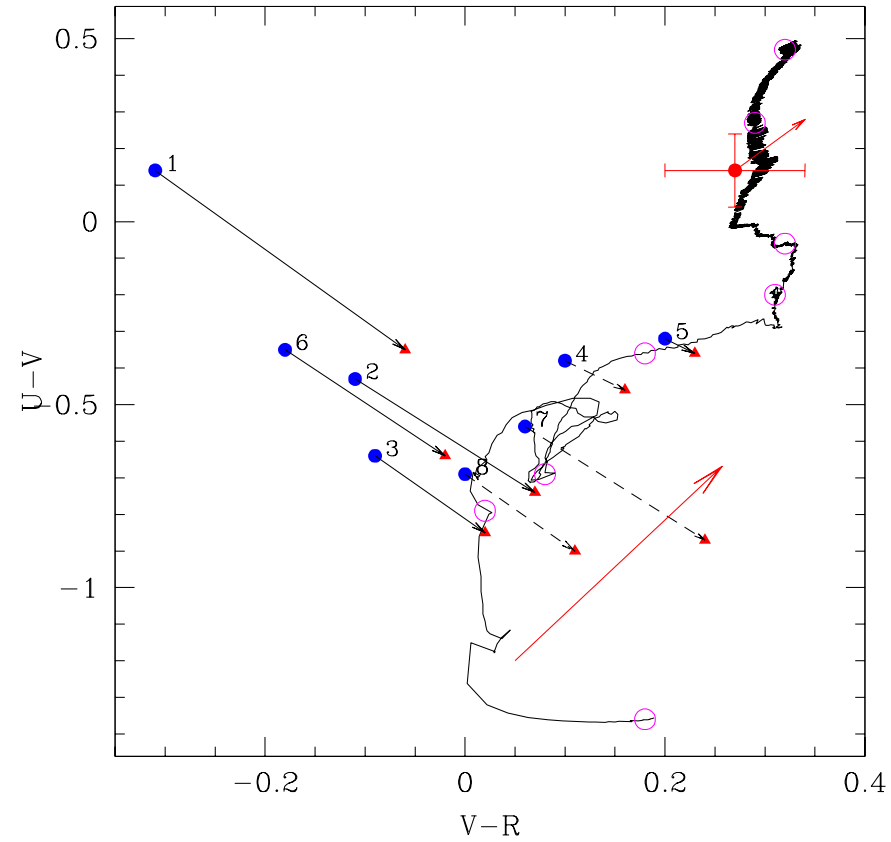

Fig. 4. $U-V / V-R$ color-color diagram of the clusters in UM 462 . Solid circles are the observed colors, triangles after the correction for the contribution of emission lines. The dashed arrows indicate when the correction was based on the $\mathrm{H} \alpha$ image rather than on spectroscopy. The dot with error bars indicates the colors of the whole galaxy and the arrow the correction for the clusters contribution. The black solid line represents the output of SB99 for an instantaneous burst of star formation, points at age 1, 5, 10, 25, 50, 100, 500 and $1000 \mathrm{Myr}$ are indicated by open circles. The vector corresponds to $1 \mathrm{mag}$. of visual extinction.

as solid lines. Despite the uncertainty the emission line correction works very well to move the observed points close to the tracks defined by the model. On those tracks the points at ages $1,5,10,25,50,100,500$ and 1000 Myr are marked by open circles with the oldest ages at the top-right of the plots.

The near-infrared data from Vanzi et al. (2002) have been used to build an optical-near-infrared color-color diagram of the clusters. This is shown in Fig. 6. The errors are larger in this case and the correction for the emission line contribution more uncertain than in the optical case. The near-infrared spectrum of Vanzi et al. (2002) in fact includes clusters \#1 only. To correct the $K$ photometry of the other clusters I have used the infrared spectrum of cluster \#1 as template and rescaled it to produce for each cluster the $\mathrm{H} \alpha / \mathrm{Br} \gamma$ ratio predicted by case $\mathrm{B}$, then used the rescaled spectrum to derive the photometric correction.

The colors of the galaxy as a whole are in all diagrams consistent with the presence of an evolved population of stars, the observed colors are plotted as solid circles with error bars, the arrows points to the colors corrected for the contribution of the clusters, they are consistent with a population older than 0.5 Gyr. Unfortunately the $K$ image is not deep enough to extract a reliable magnitude for the entire galaxy. 


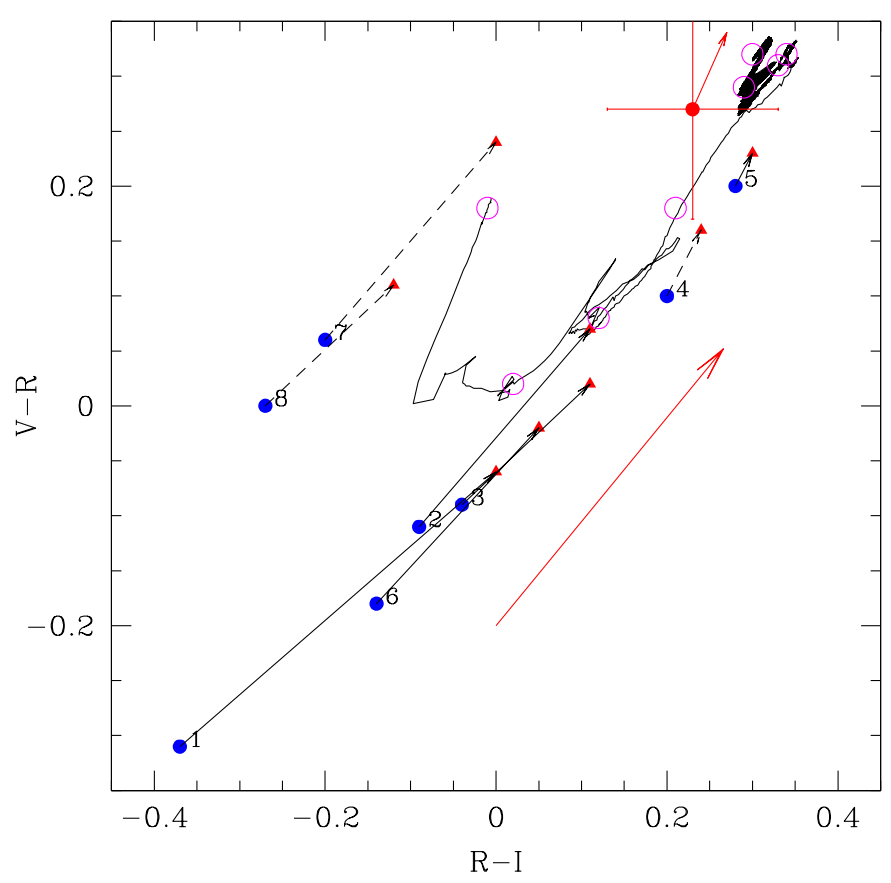

Fig. 5. Same as the previous figure for the $V-R / R-I$ diagram.

Table 3. Properties of the Clusters, the age is derived from the $E W$ of $\mathrm{H} \alpha, A_{V}$ from the Balmer decrement.

\begin{tabular}{cccccc}
\hline \hline & $M_{V}$ & $E Q W(\mathrm{H} \alpha)$ & Age $(\mathrm{Myr})$ & $A_{V}$ & $M\left(10^{5} M_{\odot}\right)$ \\
\hline 1 & -13.28 & $730 \pm 20$ & 4.7 & 0.77 & 7.2 \\
2 & -12.15 & $325 \pm 20$ & 5.1 & 0.49 & 1.2 \\
3 & -12.82 & $230 \pm 10$ & 5.8 & 0.11 & 2.1 \\
4 & -11.54 & - & - & - & - \\
5 & -11.67 & $33 \pm 5$ & 10.0 & - & 2.9 \\
6 & -11.93 & $260 \pm 10$ & 5.6 & 0.77 & 1.6 \\
7 & -11.57 & - & - & - & - \\
8 & -11.36 & - & - & - & - \\
\hline
\end{tabular}

\section{Properties of the clusters}

\subsection{Extinction}

The extinction can be estimated by the ratio $\mathrm{H} \alpha / \mathrm{H} \beta$ for the clusters with spectroscopic observations. Guseva et al. (2000) give $A_{V}=0.27$. From the fluxes of Table 2 values in the range 0.11-0.77 can be derived for cluster \#1, \#2, \#3 and \#6, in particular the highest value of extinction is obtained for cluster \#1 and \#6. The values of the extinction are listed in Table 3. For cluster \#1 the Br $\gamma$ flux of Vanzi et al. (2002) can be used in combination with $\mathrm{H} \alpha$, in this case $A_{V}=0.55$. So that the optical extinction can be considered relatively low toward all the sources observed. The contribution of the galactic extinction in the direction of UM 462, as reported by NED, is low $A_{V}=0.064$.

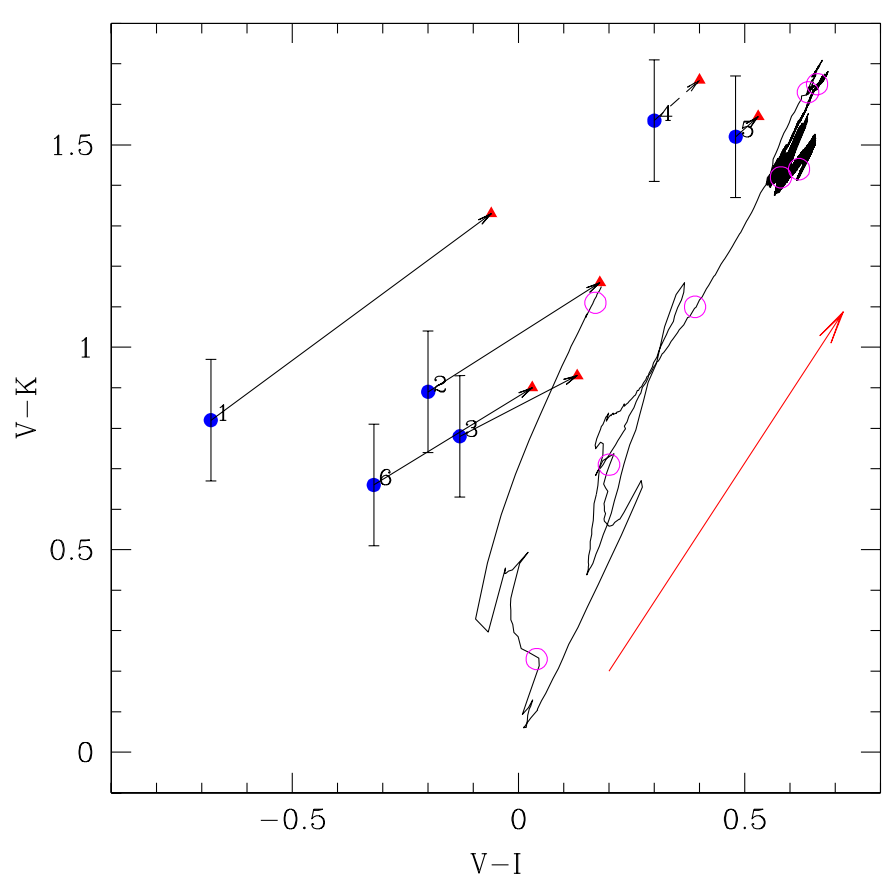

Fig. 6. Same as the previous figure for the $V-K / V-I$ diagram.

\subsection{Ages}

The stellar populations of all clusters are quite young according to their colors, typically younger than $10 \mathrm{Myr}$, as can be derived from the comparison with SB99. Clusters \#4 and \#5 are more evolved than the others. The diagram $U-V / V-R$ is quite straightforward. Clusters \#1,\#2, \#3, and \#6 show ages of about 5 Myr. The gas correction is most likely under-estimated for cluster \#1, while it could be over-estimated for clusters \#7 and \#8 which slightly deviate from the general trend. These latter clusters could also be very young objects affected by some extinction. It is difficult to disentangle the effect of extinction and age specially given the uncertainty on the corrections applied. We have seen in the previous section that there are good indications for the extinction to be low in UM 462, unfortunately no data are available to measure the extinction toward cluster \#7 and \#8. Clusters \#4 and \#5 are close to the $25 \mathrm{Myr}$ point of the model.

In the $V-R / R-I$ diagram most of the points seem to show an offset of about 0.1 mag with respect to the model, the offset however is not systematic and in particular clusters \#7 and \#8 present a trend opposite to the others so that it is difficult to give a unique interpretation. The problem could arise both from the observations and from the model. In any case clusters \#1, \#2, \#3 and \#6 are all close to 5-10 Myr, clusters \#7 and \#8 again deviates from the behavior of the other clusters and are in the very young part of the diagram, while clusters \#4 and \#5 are close to $25 \mathrm{Myr}$ with \#4 a bit younger than \#5 as it is in the previous diagram. The optical color-color diagrams then give fully consistent results.

In the $V-I / V-K$ diagram the data points show a significant offset from the model with a color excess of about $0.2-0.5$ in $V-K$. This in principle could be due to a systematic error in the $V-K$ color, however the photometry has been checked with 
the optical and $K$ photometry available in the literature both for the galaxy and for the field stars finding good agreement within the errors. Since the contribution of the underlying galaxy has been subtracted and most of the clusters have quite young ages, it seems also unlikely for the $V-K$ color to be reddened by the contribution of an evolved population. It is interesting to notice that a discrepancy similar to the one reported here is also found in the $V-I / V-K$ diagram discussed by Vanzi et al. (2000) for the metal deficient galaxy SBS 0335-052 so that it could be due to a more fundamental effect. It is well known that current stellar evolutionary tracks fail in reproducing the correct Blue to Red supergiants ratio $(B / R)$ at metallicities lower than solar (Langer \& Maeder 1995; Maeder \& Meynet 2001). In particular the number of red supergiants is highly underestimated at low metallicity. According to the calibration of Eggenberg et al. (2002) at solar metallicity $B / R=3$ while for $1 / 5 Z_{\odot} B / R=0.27$. This is mainly due to the longer duration of the red phase during the He burning. While solar single stellar population models are dominated by red supergiants stars from 6.5 to about $25 \mathrm{Myr}$ with a maximum at $10 \mathrm{Myr}$, subsolar model don't. Theory and observations agree pointing to an even longer red supergiants phase at subsolar abundance. This has been quantified by Origlia et al. (1999) unfortunately these authors did not model the $V-I$ and $V-K$ colors, however the evolution of $J-K$ which they calculate is quite significant. Standard models predict a maximum in $J-K$ around $10 \mathrm{Myr}$ which rapidly declines at $13-15 \mathrm{Myr}$, the maximum is much more prominent in solar than in subsolar models. Adjusting the red supergiants temperature and lifetime during the He burning makes $J-K$ redder and almost constant beyond $25 \mathrm{Myr}$. Since all clusters observed are in the right age interval the effect described could well explain the discrepancy of the data respect to the model. Despite the systematic deviation from the model again clusters \#1, \#2, \#3 and \#6 are close to each other in the young part of the diagram while clusters \#4 and \#5 show more evolved colors. It is interesting to notice that, beside $\mathrm{K}$, the effect must be visible in the $I$ filter so that it can possibly be responsible for the red eccess observed for some of the clusters in the $V-R / R-I$ diagram.

The age of the clusters can also be estimated in an independent way by the comparison of the equivalent width $(E W)$ of $\mathrm{H} \alpha$ with the predictions of SB99. The ages derived with this method are listed in Table 3. Clusters \#1, \#2, \#3 and \#6 have ages between 4.7 and 5.8 Myr which are fully consistent with the values derived from the stellar continuum. Cluster \#5 instead looks significantly younger in $\mathrm{H} \alpha$ than in the continuum and right at the maximum of the red supergiants phase, a fact that could explain the discrepancy for what has been said before. For cluster \#1, the only one with a NIR spectrum, the age can also be derived from $\mathrm{Br} \gamma$, the value obtained is 4.9 Myr, fully consistent with the previous estimate. From the previous analysis no obvious correlation can be found between the age of the clusters and their extinction.

The sources detected in $\mathrm{H} \alpha$ which are faint in the continuum must have a very high $E W$, they can easily be clusters younger than about 4.0 Myr. For these ages in fact SB99 predicts $\log E W(\mathrm{H} \alpha)>10^{3} \AA$. The $\mathrm{H} \alpha$ luminosities of these sources are in the range 1.4-5.4 $10^{38} \mathrm{erg} \mathrm{s}^{-1}$ that is also compatible with them being SuperNova Remnants younger than about 2500 days (Turatto et al. 1993). This latter hypothesis seems less likely since there are no reports of SN found in the galaxy during the last 15 years.

It must be noted that the tracks used for deriving the ages of the clusters both from the colors and from $\mathrm{H} \alpha$ have a metallicity slightly higher than UM 462 . We can use the tracks at $1 / 20 Z_{\odot}$ to qualitatively estimate the effect. The differences introduced even at this very low metallicity are small and certainly negligible for the present discussion.

\subsection{Luminosity and mass}

Billett et al. (2002) define as Super Star Clusters (SSC) those clusters with $M_{V}<-10.5$ at a fiducial age of 10 Myr. Such an age seems a good approximation for most of the clusters observed in UM 462. Using a distance module 30.92 (Vanzi et al. 2002) and the $V$ magnitude observed, all clusters qualify as SSCs in agreement with the finding of Vanzi et al. (2002). If the clusters were younger than $10 \mathrm{Myr}$, as it is possibly the case for cluster \#1, \#2, \#3 and \#6, the error introduced would be small, less than about $0.5 \mathrm{mag}$, and in both directions as evidenced by the evolution of $M_{V}$ with time calculated by SB99. If instead the clusters were older than $10 \mathrm{Myr}$, as cluster \#4 and \#5 are, then the brightness derived would have to be considered as a lower limits since $M_{V}$ increases steeply with time. The number of compact sources observed in UM 462 is very high compared to the size of the galaxy. If we use the specific frequency defined by Östlin (2000) $S_{11}=N_{11} \times 10^{0.4\left(M_{V, \text { host }}+15\right)}$ where $N_{11}$ is the number of objects with $M_{V} \leq-11$ and $M_{V \text {, host }}$ is the total $V$ absolute magnitude of the host galaxy we obtain $S_{11}=2.46$ which is the highest value when compared to the list of Östlin.

The total star formation taking place in the clusters can be derived from the $\mathrm{H} \alpha$ flux, summed over the sources detected. Using the calibration of Kennicutt (1998) the total star formation rate is $0.05 M_{\odot} / y r$. This value is in perfect agreement with the star formation rate of $0.06 M_{\odot} / \mathrm{yr}$ derived by Vanzi et al. (2002) on the basis of the IRAS fluxes confirming the low value of the extinction observed and also telling us that most of the star formation in UM 462 occurs in the clusters observed. The value instead contrasts with the $1.4 M_{\odot} / \mathrm{yr}$ that are obtained using the total $\mathrm{H} \alpha$ flux measured by Guseva et al. (2000). The total $\mathrm{H} \alpha$ flux summed over all the point like sources detected is $2.4 \times 10^{-13} \mathrm{erg} / \mathrm{s} / \mathrm{cm}^{2}$ which compares well with the total flux of $3.2 \times 10^{-13} \mathrm{erg} / \mathrm{s} / \mathrm{cm}^{2}$ measured by Terlevich et al. (1991), while the value of Guseva et al. (2000) is higher by more than one order of magnitude. The mass can be tentatively derived comparing the luminosity of each cluster, corrected for the extinction, with the value predicted by SB99 for the corresponding age. In deriving the mass of the clusters the IMF of SB99 that is truncated at a lower mass of $1 M_{\odot}$ has been extended to a more realistic lower cut-off of $0.1 M_{\odot}$. The masses calculated in this way are listed in Table 3. For cluster \#5 that has a quite uncertain measure of the extinction, due to the low fluxes in $\mathrm{H} \alpha$ and $\mathrm{H} \beta$ the same extinction as for clusters \#1 and \#6 has been assumed. 


\subsection{Size and morphology}

The observations have been obtained under and average seeing of $1^{\prime \prime}$ in $V$ and $0.8^{\prime \prime}$ in $I$. All clusters are barely resolved in the images indicating sizes of the order of few $10 \mathrm{pc}$. Only cluster \#3 is well resolved with a deconvolved size of $4.8^{\prime \prime}$ or more than $300 \mathrm{pc}$. This indicates that rather than single star clusters we could be observing large associations, in fact typical sizes for SSCs are of the order of few parsecs (O'Connell et al. 1995). This also means that the specific frequency derived in the previous section must be considered as an upper limit.

It is very interesting to notice that the main SSCs observed are distributed around two ring-like structures. One of them, delimited by clusters \#5 and \#6 on the north-est side, is quite regular with a radius of about 1.5 arcsec corresponding to $100 \mathrm{pc}$. The second one, surrounded by clusters \#1, \#2, \#3, \#4 and \#6, is more elongated with semiaxis of about 2.5 and 4 arcsec equivalent to 166 and $266 \mathrm{pc}$. Structures like these are not unusual in BCD galaxies, they are typically interpreted as super-bubbles driven by $\mathrm{SNe}$ generated in previous SB episodes (Martin 1998). The typical expansion velocities measured for these bubbles are of the order of few $10 \mathrm{~km} \mathrm{~s}^{-1}$ (Martin 1998). In the case of UM 462 an expansion velocity of $10 \mathrm{~km} \mathrm{~s}^{-1}$ would give an age of about $10 \mathrm{Myr}$ for the smallest bubble and about $20 \mathrm{Myr}$ for the largest one. These times are perfectly consistent with the idea that the current star formation and morphology of UM 462 are the consequence of the compression of the ISM produced by a previous episode of star formation. In this case in fact we expect the expansion age of the bubbles to be greater than the age of the clusters as it is indeed observed.

\section{Conclusions}

1. High quality optical data of the BCD galaxy UM 462 have been presented. The broad band images reveal several compact sources and two large bubbles. A narrow band image in $\mathrm{H} \alpha$ evidences the presence of further compact sources.

2. Their luminosities qualify all compact sources observed as young Super Star Clusters or very large HII associations. The colors of the stellar continuum and the $E W$ of $\mathrm{H} \alpha$ and $\mathrm{Br} \gamma$ all give fully consistent ages between few and about $25 \mathrm{Myr}$. The masses are in the range 1.2-7.2 $\times$ $10^{5} M_{\odot}$.

3. The number of clusters observed, once normalized to the luminosity of the galaxy, is very high, even compared to the most extreme cases known.

4. The extinction observed in UM 462 is low. Most of the star formation occurring in the galaxy is concentrated in the clusters observed adding up to a total value of $0.05 M_{\odot} / \mathrm{yr}$.

5. The galaxy as a whole has evolved colors indicating the presence of an underlying evolved stellar population older than 0.5 Gyr.
6. The bubbles observed are interpreted as produced by thecompression of the ISM due to a previous SB episode. The current star formation would have been triggered by this compression. The size of the bubbles and the ages of the SSCs are consistent consistent with this idea.

Acknowledgements. I wish to thank L.-M. Cairos for making her images of UM 462 available to me. I am grateful to M. Sterzik for support during the observations, to G. Hau for providing useful information during the reduction of the data and to V. Ivanov, M. Sauvage and A. Maeder for useful discussions during the preparation of this paper. L. Guzzo provided the data to remove the fringes in the $i$ band images. Finally I thank the director of La Silla, J. Melnick, for granting the observation time for this work and the anonymous referee for useful comments which contributed to improve the paper.

This research has made use of the NASA/IPAC Extragalactic Database (NED) which is operated by the Jet Propulsion Laboratory, California Institute of Technology, under contract with the National Aeronautics and Space Administration.

\section{References}

Billett, O. H., Hunter, D. A., \& Elmegreen, B. G. 2002, AJ, 123, 1454 Eggenberg, P., Meynet, G., \& Maeder, A. 2002, A\&A, 386, 576

Cairos, L. M., Vilchez, J. M., Gonzalez-Perez, J. N., et al. 2001, ApJS, 133,321

Gondhalekar, P. M., Johansson, L. E. B., Brosh, N., Glass, I. S., \& Brinks, E. 1998, A\&A, 335, 152

Guseva, N. G., Izotov, Y. I., \& Thuan, T. X. 2000, ApJ, 531, 776

Hamuy, M., Walker, A. R., Suntzeff, N. B., et al. 1992, PASP, 104, 533

Hamuy, M., Suntzeff, N. B., Heathcote, S. R., et al. 1994, PASP, 106, 566

Izotov, Y. I., \& Thuan, T. X. 1998, ApJ, 500, 188

Kennicutt, R. C. Jr. 1998, ARA\&A, 36, 189

Langer, N., \& Maeder, A. 1995, A\&A, 297, 483

Landolt, A. U. 1992, AJ, 104, 340

Leitherer, C., Schaerer, D., Goldader, J. D., et al. 1999, ApJS, 123, 3

Macalpine, G. M., \& Williams, G. A. 1983, ApJS, 45, 113

Maeder, A., \& Meynet, G. 2001, A\&A, 373, 555

Masegosa, J., Moles, M., \& Campos-Aguilar, A. 1994, ApJ, 420, 576

Martin, C. L. 1998, ApJ, 506, 222

O'Connell, R. W., Gallagher, J. S., Hunter, D. A., \& Colley, W. N. 1995, ApJ, 446, L1

Östlin, G. 2000, in Massive Stellar Clusters, Astron. Soc. Pac. Conf. Ser.

Origlia, L., Goldader, J. D., Leitherer, C., Schaerer, D., \& Oliva, E. 1999, ApJ, 514, 96

Jarret, T. H. 2000, PASP, 112, 1080

Terlevich, R., Melnick, J., Masegosa, J., Moles, M., \& Copetti, M. V. F. 1991, A\&AS, 91, 285

Turatto, M., Cappellaro, E., Danziger, I. J., et al. 1993, MNRAS, 262, 128

van Zee, L., Skillman, E. D., \& Salzer, J. J. 1998, AJ, 116, 1186

Vanzi, L., Hunt, L. K., Thuan, T. X., \& Izotov, Y. I. 2000, A\&A, 363, 493

Vanzi, L., Hunt, L. K., \& Thuan, T. X. 2002, A\&A, 390, 481 